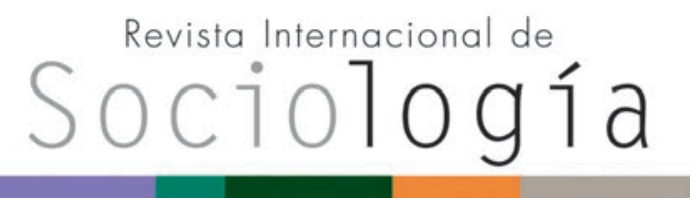

Revista Internacional de Sociología RIS

vol. 77 (3), e135, julio-septiembre, 2019, ISSN-L:0034-9712 https://doi.org/10.3989/ris.2019.77.3.18.003

\section{LA CEGUERA AL GÉNERO EN EL CURRÍCULUM DE LA CIENCIA POLÍTICA Y SU IMPACTO EN EL ALUMNADO}

\author{
TÀnia Verge Mestre \\ Universitat Pompeu Fabra \\ tania.verge@upf.edu \\ https://orcid.org/0000-0003-3798-2821
}

\author{
Alba Alonso Álvarez \\ Universidade de Santiago de Compostela \\ a.alonso@usc.es \\ https://orcid.org/0000-0001-6917-3987
}

Cómo citar este artículo / Citation: Verge Mestre, T. y A. Alonso Álvarez. 2019. "La ceguera al género en el currículum de la ciencia política y su impacto en el alumnado". Revista Internacional de Sociología 77(3):e135. https://doi.org/10.3989/ris.2019.77.3.18.003

\section{GENDER BLINDNESS IN THE POLITICAL SCIENCE CURRICULUM AND ITS IMPACT ON STUDENTS}

Copyright: (c) 2019 CSIC. Este es un artículo de acceso abierto distribuido bajo los términos de la licencia de uso y distribución Creative Commons Reconocimiento 4.0 Internacional (CC BY 4.0).

Recibido: 08/01/2018. Aceptado: 15/11/2018.

Publicado online: 24/09/2019

\section{ABstract}

Despite the prolific normative framework on gender mainstreaming in academic institutions, its implementa-tion has not been effective. This article pays attention to the Political Science curriculum. First, we examine the syllabi of first-year courses of all the Political Science degrees offered in Spain through several quantitative indicators and carry out a qualitative content analysis of the main introductory textbooks. Second, we evaluate the impact of such pervasive gender-blind curriculum on students, by means of the case study of a single university, through focus groups. The existing gap be-tween the normative framework on gender mainstream-ing in universities and the practice of its implementa-tion, specifically as regards the curriculum, allows us to reflect on how this deficit can limit the development of students' critical thinking and make their future professional practice reproduce gender inequalities.

\section{KEYWORDS}

Curriculum; Equality; Gender perspective; Teaching; Universities.

\section{Palabras Clave}

Currículum; Docencia; Igualdad; Perspectiva de género; Universidades. 


\section{INTRODUCCIÓN}

La transversalidad de la perspectiva de género, como estrategia para promover la igualdad efectiva de mujeres y hombres, ha sido recogida a nivel normativo como un principio de actuación que obliga a los poderes públicos a tener en cuenta las necesidades e intereses tanto de las mujeres como de los hombres en todas las estructuras, procesos y políticas y a trabajar para eliminar las barreras y los mecanismos de exclusión, a menudo invisibles, que sostienen la desigualdad de género (Consejo de Europa 1998). En la práctica, no obstante, su implementación se ve obstaculizada por las resistencias que genera la modificación de rutinas, procesos y prácticas enraizadas en la cultura organizativa de las propias administraciones (Cavaghan 2017; Lombardo y Mergaert 2013), tal como ocurre en la educación superior.

A pesar del prolífico marco normativo sobre perspectiva de género en las instituciones académicas (Alonso 2015), siguen operando numerosos sesgos de género en las prácticas de contratación y promoción (Izquierdo 1999; Mason y Goulden 2004), en la evaluación de la productividad académica (Izquierdo, León y Mora 2008; Wenneràs y Wold 1997), en la asignación de responsabilidades de dirección y de gestión (Castaño, Vázquez-Cupeiro y Martínez-Cantos 2017; Monroe et al. 2008), o en la docencia y la investigación (Grünberg 2011). ${ }^{1}$ Este artículo se centra en los déficits en la implementación de la perspectiva de género en el currículum, concretamente en los estudios de Ciencia Política.

El objetivo del artículo es doble. En primer lugar, exploramos hasta qué punto el currículum de la Ciencia Política incorpora el conocimiento en materia de desigualdades entre mujeres y hombres y recoge las contribuciones de los estudios de género. Para ello, examinamos las guías docentes de primer curso de todos los grados de Ciencia Política impartidos en las universidades españolas, ${ }^{2}$ así como los manuales introductorios citados con mayor frecuencia en la bibliografía de las guías docentes. En segundo lugar, evaluamos el impacto sobre el alumnado de la ausencia de perspectiva de género en las guías y en los manuales, mediante el estudio de caso de una universidad, a través de grupos de discusión. La brecha detectada entre el marco normativo sobre transversalidad de género y la práctica de su implementación en la docencia universitaria, concretamente en el currículum, nos permite reflexionar sobre cómo este déficit puede limitar el desarrollo del pensamiento crítico del alumnado y hacer que su futura práctica profesional reproduzca la desigualdad de género.

\section{MARCO NORMATIVO Y DÉFICITS DE IMPLEMENTACIÓN DE LA TRANSVER- SALIDAD DE GÉNERO}

Desde los años 90, se han desarrollado en España varios instrumentos para la implementación de la transversalidad de género (informes de impacto de género, planes de igualdad, formación especializada, etc.). En el ámbito universitario, ha sido clave el impulso de la Comisión Europea como pionera en la inclusión de criterios con perspectiva de género en los programas-marco de apoyo a la investigación (Comisión Europea 2000; Alonso 2015), tales como el equilibrio por sexos de los equipos, la revisión del androcentrismo de la producción científica y el llamamiento a cubrir las lagunas en el conocimiento sobre las mujeres y las desigualdades de género a través de investigaciones especializadas. Asimismo, en la transición hacia el Espacio Europeo de Educación Superior, el Plan Bolonia incorporó el mandato de incluir los estudios de género en la docencia universitaria (Kortendiek 2011).

Influido por el enfoque europeo, el marco normativo español ha desplegado esta perspectiva en la educación superior. La Ley orgánica 3/2007, para la igualdad efectiva de mujeres y hombres, establece la obligatoriedad de que los planes de estudio incorporen el significado y alcance de la igualdad de género y promueve el desarrollo de grados y posgrados especializados (artículo 25). Estas disposiciones han sido recogidas también por normas autonómicas análogas. La Ley 14/2011, de la Ciencia, la Tecnología y la Innovación, por su parte, impulsa la introducción del género como una categoría transversal en la investigación y la promoción de los estudios de género (artículos 2.k, 33.j y disp. ad. 13. ${ }^{a}$ ). En esta misma línea, el Libro Blanco de ANECA para la Titulación de Ciencias Políticas (2005) incluye como competencia el conocimiento de los componentes básicos de las desigualdades sociales y menciona las políticas de igualdad como una de las principales orientaciones profesionales.

La transversalidad de género también viene impulsada por las asociaciones profesionales. Ya en 1991 la American Political Science Association (APSA) definía su aplicación en la docencia de la siguiente forma: "que el carácter y las implicaciones de la diversidad étnica, de género y cultural y las dimensiones internacionales y transnacionales de los problemas y las políticas se trabajen en todos los cursos relevantes de manera transversal ['mainstreamed', en inglés] - rehuyendo un tratamiento separado y único en uno o dos cursos específicos" (Wahlke 1991: 53, énfasis en el texto original). La Asociación Española de Ciencia Política (AECPA) ha incorporado recientemente a sus estatutos el compromiso de "desempeñar todas las actividades con especial atención a la promoción y visibilidad de la perspectiva de género en la docencia y la investigación" (artículo 4.9). Asimismo, varias revistas académicas han dedicado monográficos a esta cuestión, como PS: Political Science \& Politics (49:3, 2016), European Political Science $(15: 3,2016)$ o Politics \& Gender (9:2, 2013). 
Una docencia con perspectiva de género estimula el pensamiento crítico, proporcionando al alumnado nuevas herramientas para identificar las normas y los roles de género subyacentes a la sociedad, la política y la economía y para problematizar los patrones de socialización dominantes (Lyle-Gonga 2013). Además, incluir las experiencias de las mujeres y reconocer sus contribuciones puede empoderar a las estudiantes, acercarlas a la disciplina y animarlas a proseguir una carrera académica (Cassese y Bos 2013). Cabe recordar que, mientras en 1998 las mujeres representaban en España el 55 \% del alumnado de Ciencias Políticas, actualmente constituyen el 45 \% (Elizondo 2015). La adopción de la perspectiva de género comporta igualmente beneficios directos sobre la calidad de la docencia, tanto para el alumnado como para el profesorado. En primer lugar, permite reconocer que la histórica desigualdad entre mujeres y hombres ha moldeado la propia conceptualización de la 'política' (Matthes 2013: 237), poniendo de manifiesto que aquello concebido como conocimiento neutral y objetivo no deja de ser el resultado de un sistema de relaciones de poder entre diferentes categorías sociales, entre ellas el género (Grünberg 2011: 7). En segundo lugar, las contribuciones epistemológicas, teóricas y empíricas producidas por los estudios que adoptan esta mirada mejoran la calidad de las asignaturas (Mertus 2007). Concretamente, el conocimiento sobre la incidencia de las desigualdades en la vida pública y en la vida privada evita cometer errores de conceptualización teórica, modelización o análisis fruto del uso indistinto de las variables 'sexo' y 'género' (Lovenduski 1998).

Sin embargo, el marco normativo y las recomendaciones de las asociaciones profesionales presentan un déficit de implementación importante en este campo. Como señala la literatura especializada, las políticas de igualdad de género suelen ir acompañadas de resistencias, tanto en su adopción como en su implementación (Lombardo y Mergaert 2013; Verloo 2018). En el caso de la implementación de la transversalidad de género en la docencia, se detectan resistencias indirectas al cambio, en forma de inacción, esto es, de ausencia de decisiones y de falta de recursos e incentivos adecuados. Por un lado, las agencias evaluadoras no han elaborado indicadores ni criterios de calidad en esta materia para supervisar el cumplimiento de la normativa en el desarrollo curricular de los programas de grado y de postgrado (véase Nuño Gómez y Álvarez Conde 2017: 284-86). ${ }^{3}$ Por otro lado, la formación continua proporcionada por las universidades no cuenta con una oferta estable ni suficiente de cursos sobre este enfoque, lo que impide que el profesorado adquiera las competencias necesarias para aplicarlo y provoca que no lo perciba como necesario o lo considere una carga extra de trabajo (Verge y Cabruja 2017). También se ha constatado la existencia de resisten- cias directas, como la predominante consideración del mandato legal de la transversalidad de género como una cuestión ideológica que supuestamente infringiría tanto la autonomía universitaria como la libertad de cátedra del profesorado. A su vez, tiende a negarse la relevancia del género para el contenido curricular, e incluso se considera la desigualdad entre mujeres y hombres un aspecto ya superado (véase Verge, Ferrer-Fons y González 2018).

Tales inacciones y resistencias activas han dado como resultado que, hoy en día, en España los grados carezcan en buena medida de contenidos curriculares especializados o con perspectiva de género (Verge y Cabruja 2017). Fundamentalmente, son las unidades de igualdad de las universidades quienes suplen este déficit formativo e impulsan la transversalidad de género tanto en la docencia como en el resto de políticas universitarias, mediante las acciones incluidas en el respectivo plan de igualdad. Con todo, al no gozar de atribución competencial en materia docente y carecer de incentivos institucionales y de supervisión efectiva sobre su implementación, su efectividad se ha visto limitada (Nuño Gómez y Álvarez Conde 2017: 288-89).

En el caso de la docencia de la Ciencia Política en España, los cambios curriculares han sido escasos (Foster et al. 2013; Mügge, Evans y Engeli 2016). Determinadas características de esta disciplina dificultan la implementación de la perspectiva de género. Además de persistir una relativa marginalización de las mujeres en la profesión (Bates, Jenkins y Pfaelger 2012; Elizondo 2015; TollesonRinehart y Carroll 2006), se sigue otorgando un estatus secundario a los estudios de género (Atchison 2013: 228; Valiente 2002: 770), lo que comporta la (re)producción de un conocimiento politológico basado en una rígida separación entre vida pública y vida privada que considera a los hombres como los actores políticos por antonomasia (Foster et al. 2013; Lovenduski 1998).

\section{DATOS Y METODOLOGÍA}

De los distintos aspectos de la docencia, en este artículo nos centramos en el contenido curricular por su impacto directo sobre la formación del alumnado. En primer lugar, para medir hasta qué punto la enseñanza de la Ciencia Política incorpora la perspectiva de género, se analizaron las guías docentes de las 171 asignaturas de primer curso de todos los grados en Ciencia Política impartidos en las universidades españolas (18 en total) del curso académico 20152016. La idoneidad de esta unidad de análisis radica en que las materias de primer curso suelen tener un carácter introductorio, presentando los principales temas y enfoques que integran el núcleo duro de la disciplina. Como indican Cassese y Bos (2013: 217), "el contenido que se excluye revela tanto de la disci- 
plina como el que se incluye". Estas asignaturas proporcionan, pues, una buena aproximación para valorar hasta qué punto los planes de estudio incorporan la perspectiva de género de manera transversal, más allá de la inclusión de materias especializadas en género ofertadas en cursos más avanzados (generalmente, asignaturas optativas). Para cada una de las guías docentes se han analizado los siguientes indicadores ${ }^{4}$

- Contenidos: Presencia de temas relativos a la desigualdad de género, a la situación de las mujeres o a la interseccionalidad del género con otros ejes de subordinación (clase social, raza, opción sexual o diversidad funcional).

- Competencias: Inclusión de competencias (cognitivas, actitudinales o instrumentales) relacionadas con la igualdad de género o con la capacidad de analizar diferentes temáticas desde la perspectiva de género.

- Referencias: Porcentaje de autoras en la bibliografía básica -denominada en algunas guías como obligatoria - y modo en que las obras son citadas (nombre completo o iniciales), así como referencia a manuales especializados, como el Oxford Handbook of Gender and Politics (Waylen et al. 2013) o el manual Ciencia Política con Perspectiva de Género (Lois y Alonso 2014a)

Simultáneamente, siendo común el uso de manuales en las asignaturas introductorias de primer curso, se examinaron aquellos más frecuentemente citados en la bibliografía (obligatoria o recomendada) de las guías docentes, de entre los escritos en castellano. Se trata de siete obras publicadas entre 1996 y 2016 , como muestra la tabla 1 . La presencia de mujeres como autoras, editoras o colaboradoras de estos manuales es escasa. Al no existir una versión electrónica de todos ellos, no fue posible recontar la aparición de palabras clave. De manera alternativa, siguiendo a Atchison (2017), se realizó un análisis de contenido cualitativo de los manuales basado en los siguientes aspectos: definición del concepto género; grado de transversalidad del género en los distintos capítulos y uso analítico del mismo; referencia a las mujeres como protagonistas de la vida política, y reconocimiento de las obras producidas por mujeres.

En segundo lugar, puesto que cabría la posibilidad de que en las guías docentes no apareciera de manera explícita la perspectiva de género pero sí fuera trabajada en el aula mediante distintas actividades docentes, la información obtenida mediante el análisis de las guías y los manuales se ha triangulado con grupos de discusión con estudiantes. Para realizar esta comprobación, nos centramos en un solo caso, el Grado en Ciencia Política y de la Administración de la Universitat Pompeu Fabra (Barcelona), una universidad pública joven —creada en 1990 - que imparte estos estudios desde el curso 1995-1996 (como licenciatura hasta el curso 2008-2009). Empleamos el análisis de este grado como un "estudio de caso instrumental" (Stake 1999: 17), para investigar en detalle el impacto sobre el alumnado de su (falta de) exposición a contenidos de género. Se ha comprobado que no se trata de un caso outlier ni en sentido positivo ni en sentido negativo, encontrándose los resultados de este grado sobre la media de los resultados del resto de grados en cuanto a contenidos curriculares relacionados con el género (véase el apéndice).

La media de estudiantes mujeres en el Grado en Ciencia Política y de la Administración de la UPF en los últimos siete años se ubica en el $44,8 \%$, y la media de docentes mujeres en el 37,5\%. ${ }^{5}$ Tras completar el cuarto año de implantación del Plan Bolonia, se recogió información acerca de la incorporación de la perspectiva de género en el conjunto de asignaturas a través de un proyecto de innovación docente (véase Verge, Ferrer-Fons y González, 2017), por lo que disponemos de información no solo del primer curso, sino del grado entero. El grado contó inicialmente con una asignatura optativa centrada en estudios de género, incorporándose en el curso académico 2014-2015 una segunda asignatura especializada.

Tabla 1.

Manuales de Ciencia Política analizados

\begin{tabular}{|c|c|c|}
\hline Referencia ( $1^{\text {a }}$ edición) & N. ${ }^{\circ}$ capítulos & N. ${ }^{\circ}$ de autores y autoras \\
\hline - Miquel Caminal (ed.) (1996) Manual de Ciencia Política. Madrid: Tecnos. & 21 & 20 hombres, 1 mujer \\
\hline - Rafael del Águila (ed.) (1997) Manual de Ciencia Política. Madrid: Trotta. & 19 & 16 hombres, 2 mujeres \\
\hline - Josep Ma Vallès (2000) Ciencia política: una introducción. Barcelona: Ariel. & 27 & 1 hombre \\
\hline $\begin{array}{l}\text { - Michael J. Sodaro (2006) Política y Ciencia Política: una introducción. Madrid: } \\
\text { McGraw-Hill. }\end{array}$ & 17 & $\begin{array}{l}1 \text { autor principal con colaboración } \\
\text { de } 3 \text { hombres y } 2 \text { mujeres }\end{array}$ \\
\hline - Josep M. Colomer (2009) Ciencia de la política. Barcelona: Ariel. & 16 & 1 hombre \\
\hline - Edurne Uriarte (2010) Introducción a la Ciencia Política. Madrid: Tecnos. & 17 & 1 mujer \\
\hline $\begin{array}{l}\text { - Mikel Barreda y Leticia M. Ruiz Rodríguez (coord.) (2016) Análisis de la Po- } \\
\text { lítica. Enfoques y herramientas de la Ciencia Política. Barcelona: Huygens. }\end{array}$ & 25 & 21 hombres, 10 mujeres \\
\hline
\end{tabular}

Fuente: Elaboración propia. 
Entre enero y febrero de 2014 se realizaron tres grupos de discusión con estudiantes de segundo, tercero y cuarto curso — descartando al alumnado de primero por no disponer de suficiente perspectiva del grado-. Los grupos duraron 90 minutos e incluyeron entre seis y ocho estudiantes. Dos fueron mixtos y uno no mixto (solo chicas). ${ }^{6}$ La discusión partió de un cuestionario semi-estructurado. La convocatoria se publicitó mediante carteles, anuncios virtuales y avisos en clase. Para evitar la auto-selección de participantes y el sesgo de deseabilidad social en las respuestas, los grupos se anunciaron como una discusión sobre la calidad de la docencia y la interacción profesorado-alumnado. El examen de los grupos de discusión nos permite analizar de manera exploratoria el impacto sobre el alumnado de la presencia o ausencia de la perspectiva de género en las referencias bibliográficas y en los contenidos de las guías docentes, así como en la gestión del entorno de aprendizaje por parte del profesorado.

\section{LA CEguera AL GÉNERO de LOS PLA- NES DOCENTES Y MANUALES}

Durante el curso 2015-2016, tan solo siete de los 18 grados de Ciencia Política impartidos en España ofertaban asignaturas especializadas en género y política, pese a la doble estrategia explicitada en el mandato legal: impartir asignaturas específicas e incorporar el género de manera transversal en el currículum del resto de asignaturas (Alonso y Lombardo 2016). Los grados que incluyen esta formación específica tienden a ofertar solamente una asignatura, en general optativa, lo que comporta que la mayor parte de estudiantes se gradúen sin una exposición mínima a estos contenidos. ¿Hasta qué punto se incorpora la perspectiva de género en las asignaturas?

Como muestra la tabla 2 (véanse los datos desagregados por universidad en el apéndice), tan solo el $8,8 \%$ de las 171 guías docentes de primer curso examinadas incluyen temas relativos a las desigualdades de género, a la situación de las mujeres o a la interseccionalidad, pese a que resulta muy difícil identificar un área de la Ciencia Política en la que el género no sea relevante, debido al impacto diferenciado de las estructuras y políticas públicas sobre mujeres y hombres (Waylen et al. 2013; Beckwith 2005). Los manuales especializados en género aparecen tan solo en el $2,4 \%$ de las guías (Tabla 2). En cuanto a las competencias, el $18,1 \%$ de las guías sitúa entre el conjunto de habilidades a adquirir, por ejemplo, el "compromiso con la igualdad de género" o la capacidad de "identificar y analizar críticamente la desigualdad de género y su intersección con otros ejes de desigualdad". Se trata de una cifra muy baja tratándose de asignaturas introductorias de primer curso, en que estas competencias son fundamentales para comprender la realidad política y social.
Tabla 2.

Inclusión del género en las guías docentes

\begin{tabular}{lc}
\hline & Total \\
\hline Temas relacionados con género & $8,8 \%$ \\
Manual sobre género y política & $2,4 \%$ \\
$>30 \%$ autoras en listado referencias & $13,1 \%$ \\
Nombre completo de autores y autoras & $35,4 \%$ \\
Competencias relativas a la igualdad de género & $18,1 \%$ \\
\hline
\end{tabular}

Fuente: Elaboración propia.

Nota: Los valores indican el porcentaje de asignaturas.

El análisis de la bibliografía muestra que el nombre completo de autores y autoras se proporciona en el $35,4 \%$ de las guías, lo que invisibiliza la presencia de las mujeres como productoras de conocimiento, ya que, cuando solo aparece la inicial del nombre, se tiende a considerar, por defecto, que la autoría es masculina (Bosque 2012: 3). Entre las guías que proporcionan el nombre completo, un escaso $13,1 \%$ incluyen un mínimo del $30 \%$ de autoras. Las politólogas están, por lo tanto, doblemente en minoría como referentes de la disciplina, tanto entre el profesorado, especialmente en las categorías de mayor rango, con un $7,3 \%$ de mujeres catedráticas (Elizondo 2015), como en las referencias bibliográficas. Todo ello contribuye a crear un imaginario en que los hombres simbolizan el experto por excelencia (Kantola 2008: 204) y se (re)produce una determinada asociación entre género, estatus y poder (cf. Cassese y Bos 2013: 215).

Los manuales de Ciencia Política más comunes en las guías docentes, tanto antiguos como recientes, adolecen también de una remarcable ceguera al género, ignorándose así que los estudios de género y política son uno de los campos de investigación de mayor crecimiento, como constata el hecho de que el Standing Group on Gender and Politics del European Consortium for Political Research (ECPR) sea el segundo en volumen de miembros (figura 1). Este déficit es muy trascendente, ya que los manuales ejercen de "repositorios del conocimiento oficial" (Ferree y Hall 1996). Igualmente, la ausencia generalizada de las mujeres como sujetos activos del contenido de los manuales refuerza estereotipos (por ejemplo, la idea que son menos activas en la política) e impide que las estudiantes tengan referentes más cercanos de agencia política (Atchison 2017: 195).

Por una parte, como muestra la tabla 3, solamente un manual, el editado por Barreda y Ruiz (2016), contiene un capítulo dedicado a género y política (Tabla 3). Este capítulo define el concepto 'género', presenta las teorías feministas del Estado, la interacción entre el movimiento feminista y las instituciones que desarrollan las políticas de igualdad, las estrategias políticas para promover la igualdad, incluyendo la transversalidad de género, y examina el impacto 
Figura 1

Nube de palabras según volumen de miembros, ECPR standing groups

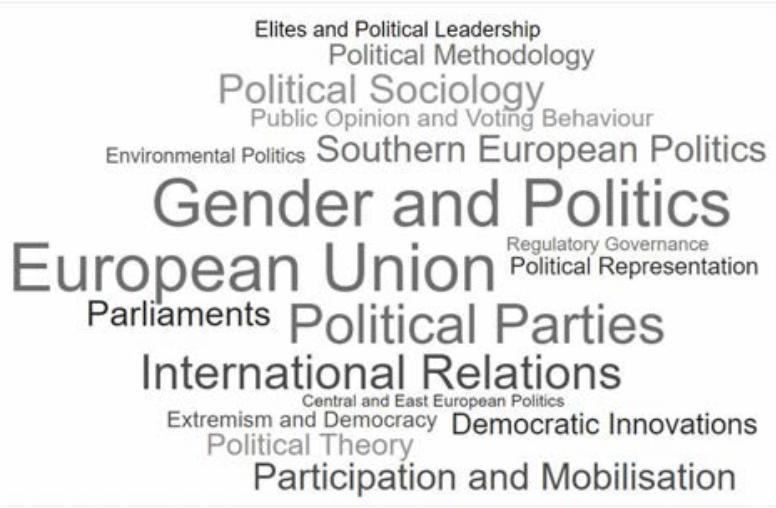

del género en la representación política. No obstante, el resto de los capítulos son fundamentalmente ciegos a esta perspectiva, con la excepción del correspondiente a metodología, que proporciona diferentes ejemplos de investigaciones sobre género y política. La gran mayoría de manuales no incluyen ni siquiera un epígrafe relativo a las mujeres, el feminismo o las políticas de igualdad. Constituyen excepciones el manual de Sodaro (2006), que define el género -aunque no se hace un uso analítico del mismo en ningún capítulo-, presenta la crítica feminista al liberalismo e incluye el feminismo como una de las principales ideologías del siglo $\mathrm{XX}^{7}$; y el de Uriarte (2010), con una sub-sección de un capítulo dedicada a las políticas de igualdad de oportunidades de la Unión Europea.

Por otra parte, tan solo el manual de Vallès (2000) hace mención a las mujeres y a las desigualdades de género a lo largo del texto, aunque sea superficialmente y sin recoger las aportaciones de los estudios de género a la disciplina. El resto de manuales no transversaliza estos contenidos en los distintos capítulos, ni las menciones a las mujeres superan una línea de texto. La mención más recurrente es a la extensión del sufragio femenino o al movimiento feminista, dedicándose desde una frase a un breve párrafo a los mismos. El feminismo como teoría política, con las excepciones ya comentadas de Sodaro (2006) y Barreda y Ruiz (2016), está ausente, pese a contar con un corpus de conocimiento de más de tres siglos de trayectoria.

Las mujeres no aparecen casi en los manuales, asumiendo que los actores, las instituciones, los procesos y las políticas públicas son neutrales al género. Se perpetúa, así, la representación del hombre como categoría universal. A excepción del manual de Vallès (2000), es remarcable la nula discusión sobre la importancia del género para explicar las diferencias en el comportamiento político, a pesar de haberse demostrado que las variaciones en la implicación política radican en la asimetría de recursos generados en el ámbito privado, a partir de la imperante división sexual de trabajo que proporciona a los hombres más tiempo libre y mayores posibilidades de interacción social (Verge 2014).

Aunque el género tiene un impacto relevante sobre las instituciones y las estructuras políticas (Lois y Alonso 2014b), la infra-representación política de las mujeres tampoco aparece en la mayoría de manuales o lo hace de manera muy limitada en Uriarte (2010), del Águila (1997) y Sodaro (2006), además de Barreda y Ruiz (2016) —en el capítulo sobre género y política, pero no en los relacionados con instituciones o representación-. La adopción de cuotas electorales, la reforma electoral más extendida a nivel mundial, se recoge superficialmente en Sodaro (2006) y Vallès (2000), sin mención a sus distintas modalidades o a su encaje en los distintos sistemas electorales.

Tabla 3.

El género de los manuales

\begin{tabular}{|c|c|c|}
\hline Manual & Contenido de género & Autoras en la bibliografía \\
\hline Caminal (1996) & Ninguno. & $5,3 \%$ \\
\hline del Águila (1997) & Ninguno. & $5,0 \%$ \\
\hline Vallès (2000) & $\begin{array}{l}\text { Hace referencia a las mujeres a lo largo del manual, aunque de manera } \\
\text { superficial. }\end{array}$ & $7,5 \%$ \\
\hline Sodaro (2006) & $\begin{array}{l}\text { Un capítulo define el género; otro capítulo presenta la crítica feminista al } \\
\text { liberalismo; y otro capítulo incluye el feminismo como una de las grandes } \\
\text { ideologías del siglo XX. }\end{array}$ & $8,2 \%$ \\
\hline Colomer (2009) & Ninguno. & $3,0 \%$ \\
\hline Uriarte (2010) & $\begin{array}{l}\text { Incluye una sub-sección en un capítulo (políticas de igualdad de oportuni- } \\
\text { dades en la UE). }\end{array}$ & $8,7 \%$ \\
\hline $\begin{array}{l}\text { Barreda y Ruiz } \\
\text { Rodríguez (2016) }\end{array}$ & $\begin{array}{l}\text { Dedica un capítulo entero a la temática de Género y Política. El capítulo de } \\
\text { metodología pone ejemplos de investigaciones sobre género y política. }\end{array}$ & $18,6 \%$ \\
\hline
\end{tabular}

Fuente: Elaboración propia. 
Los manuales analizados tienden a presentar a las mujeres como actores políticos tan solo de manera indirecta, al mencionar el movimiento feminista. Las explicaciones sobre la participación política o las elites se refieren sistemáticamente a los hombres, produciendo un imaginario simbólico donde el homo politicus es masculino, sin problematizar los sesgos de género que atraviesan los partidos políticos y los movimientos sociales (véase Martínez Hernández 2014). Las mujeres y las desigualdades de género también están ausentes de los capítulos sobre el Estado de Bienestar o las políticas públicas, ignorando las más de dos décadas de aplicación de la transversalidad de género por parte de organismos internacionales y administraciones públicas (Bustelo 2014; Peterson, López y Platero 2014).

Casi todos los manuales definen la política como una relación de poder - articulación de conflictoso como una actividad de gobierno, incluyendo la provisión de servicios. Se consagra, así, la separación entre vida pública y vida privada (con la excepción del capítulo de género y política del manual de Barreda y Ruiz, 2016), pese a las numerosas contribuciones teóricas feministas que han problematizado tal división y reflexionado sobre la desigualdad de género en la producción, distribución y ejercicio del poder (véase Lois y de la Fuente 2014). Para estos manuales, lo personal sigue sin ser político, definiéndose la política bajo unos parámetros muy estrechos. Solamente el manual de Vallès (2010: 26) parte de una definición más amplia de poder, que abarca aspectos tradicionalmente considerados como privados, señalándose al movimiento feminista como responsable de la politización de la relación (de poder) desigual $-\mathrm{y}$, por ende, política- entre mujeres y hombres. La intersección del género con otros ejes de desigualdad no aparece en ningún manual. Se trata de una omisión crítica, puesto que las diferencias entre hombres y mujeres en cuanto a experiencias y acceso a recursos y oportunidades son tan relevantes como las que se dan en el seno de las categorías 'mujer' y 'hombre' debido al impacto de la edad, la clase social, la etnicidad, la orientación sexual o la diversidad funcional (cf. Lovenduski 1998: 335).

Los ejemplos utilizados en los manuales también parten a menudo de estereotipos de género. Sirva como ilustración el utilizado por Colomer (2017: 75) para explicar un dilema de acción colectiva: "el presidente de la asociación de vecinos organiza una patrulla nocturna con voluntarios — tres hombres serán convocados cada noche al azar - para dar captura a un individuo que se dedica a agredir a las mujeres que viven en un barrio residencial. Para ello manda una carta a cada cabeza de familia en la que expone el plan y asigna una ronda de patrulla a cada destinatario". Este ejemplo refuerza el rol de la mujer víctimadébil y el del hombre protagonista-agresor o fuerte.
Por último, tanto en los manuales más antiguos como en los más recientes, las referencias a investigaciones realizadas por mujeres representan un porcentaje pírrico en comparación con su peso en la profesión. Menos de la mitad de los manuales incluyen las iniciales del nombre de los y las autoras (Colomer, Uriarte y Sodaro). Las obras escritas por hombres también predominan en los recuadros con extractos de texto y citas literales destacadas para ilustrar las explicaciones: de los 106 extractos proporcionados en Colomer (2017), tan solo dos corresponden a autoras; en el caso de Uriarte (2010), son solo tres de los 35 extractos. Por último, ninguno de los manuales utiliza un lenguaje inclusivo.

\section{EL IMPACTO DE LA CEGUERA AL GÉ- NERO SOBRE EL ALUMNADO}

Para profundizar en el análisis, seguimos con el estudio de caso del Grado en Ciencia Política de la Universitat Pompeu Fabra. Según la evaluación realizada por Verge, Ferrer-Fons y González (2017), en el curso 2013-2014 tan solo el 9,3 \% de las guías docentes del conjunto del grado contenían temas de género y la presencia media de las autoras en la bibliografía era del $19 \%$. Estos resultados son similares a los presentados en el apartado anterior, indicando que la ceguera al género no es un fenómeno exclusivo del primer curso del grado. ¿Cómo impacta dicha ceguera del currículum sobre el alumnado? Damos voz aquí a su experiencia a través del análisis de los grupos de discusión con estudiantes.

\section{La brecha de género en la bibliografía}

Antes de iniciar la discusión, se propuso cumplimentar un breve cuestionario. Una de las preguntas pedía que se nombraran tres autores y tres autoras trabajados a lo largo del grado. De media, se citaron 2,78 autores y 1,50 autoras, una diferencia notable que refleja la brecha de género que presentan las guías docentes y los manuales. Descontando al profesorado citado, el número de autores reportados básicamente se mantuvo $(2,50)$, pero el de autoras cayó hasta el 0,39. La gran mayoría de estudiantes nunca se había planteado la existencia de esta brecha y se sorprendieron de que, siendo tan dramática, hubiera pasado desapercibida:

Cuando hemos escrito los nombres de los autores y tal ha sido un, vale, no tenemos autoras (...). Realmente, hemos visto muchos autores y ninguna autora (Chica, $2^{\circ}$ curso, mixto).

No le había dado muchas vueltas. Quiero decir, pensaba: 'ostras, cuántos autores, ¿no?'. Pero hasta hoy tampoco las había echado en falta. (...) Estamos en el siglo XXI y no sé nada de las mujeres; no conozco autoras... (Chica, $3 \% 4^{\circ}$ curso, mixto).

Para dar cuenta del bajo conocimiento de autoras, el alumnado recurría inicialmente como explicación 
a la discriminación histórica de las mujeres en el acceso a la universidad y planteaba que el paso del tiempo equilibraría las proporciones:

Puede ser que en su momento no hubiera mujeres que escribieran obras; quizás no han resultado tan importantes como ellos porque estaban discriminadas (Chico, $2^{\circ}$ curso, mixto).

No sé... espero que a partir de ahora, de aquí a 50 años... (Chica, $2^{\circ}$ curso, no mixto).

Este tipo de reflexión está igualmente extendida entre el profesorado, que justifica la brecha de género en las referencias bibliográficas de los artículos de investigación (Maliniak, Powersa y Walter 2013) por la supuesta baja proporción de politólogas en su campo. Investigaciones recientes muestran que la brecha es, en buena medida, el producto de un mayor uso de la auto-cita por parte de los hombres y una mayor recurrencia de estos a citar obras de otros hombres (Hakanson 2005). Por este motivo, algunas revistas académicas (como International Studies Quarterly) ya piden que se preste atención al equilibrio de género en la bibliografía de los artículos que se someten a revisión. ${ }^{8}$ Para comprobar la brecha de género en la bibliografía de sus guías docentes, el profesorado puede consultar el aplicativo online Gender Balance Assessment Tool. ${ }^{9}$

No obstante, la inercia histórica resulta una explicación insuficiente para el alumnado, generándole estupefacción no haber leído obras escritas por mujeres al tratar temas de actualidad. Además, produce indignación, especialmente entre las estudiantes, que esta diferencia no se problematice en clase:

Cuando no se trata de teoría política, cuando se explica, no sé, participación política, vemos muchos autores actuales. ¿Por qué no vemos autoras? (Chica, $3 \% 4^{\circ}$ curso, mixto).

El problema no es que se publiquen más o menos textos, sino que no te hablen de ellas [de las autoras] (Chica, 3\% $4^{\circ}$ curso, mixto).

La brecha de género en las obras destacadas a lo largo de los estudios, junto a la infra-representación de las profesoras, repercute en la propia relación profesorado-alumnado. Se reporta una menor asistencia a clase y se admite prestar menos atención cuando la asignatura es impartida por profesoras, quienes también ven más a menudo cuestionada su autoridad. La intersección de género y edad solo es negativa para las mujeres, siendo las profesoras jóvenes las más perjudicadas por estos sesgos (Arbuckle y Williams 2003; véase también Rifà-Valls y Duarte 2013). En palabras del propio alumnado:

Cuando hay un profesor hombre va más gente a clase, no creo que digan "no voy porque es una mujer", pero les impone menos (Chica, $2^{\circ}$ curso, no mixto).

A veces, una mujer, con toda la clase, como que da la sensación de menos autoridad (Chica, $2^{\circ}$ curso, no mixto).
En la misma línea, la valoración que se hace del profesorado depende de lo que se percibe como socialmente prestigioso. A su vez, se considera que el propio profesorado adopta el rol social que se le atribuye:

La mujer tiene que justificar más que el hombre el estatus de por qué está ahí (Chico, $2^{\circ}$ curso, mixto).

A la mujer se la valora por los estudios que tiene y al hombre por el simple reconocimiento, por el prestigio social que tiene, si sale en la televisión, etc. (Chico, $2^{\circ}$ curso, mixto).

De hecho, numerosos estudios muestran que las evaluaciones del profesorado están sesgadas por razón de género. Los profesores reciben sistemáticamente evaluaciones más positivas del alumnado en prácticamente todos los indicadores (MacNell, Driscoll y Hunt 2015), con la excepción de la empatía y la cercanía, típicamente asociados a las mujeres (Schmidt 2015). Con todo, las profesoras se enfrentan a un conflicto de rol, ya que el cuidado no es necesariamente valorado como un comportamiento profesional (Timperley 2013: 90). Este dilema irresoluble, según el alumnado, no afecta a los profesores:

Las profesoras tienen que ponerse en un extremo o en otro, pero los profesores no lo necesitan; no les hace falta parecer más estrictos o más cercanos (Chica, $2^{\circ}$ curso, mixto).

De este modo, tales sesgos de género deberían ser tenidos en cuenta en las evaluaciones de la docencia por parte de los centros, para evitar causar un perjuicio en la promoción de las profesoras.

\section{La ceguera al género de los contenidos}

No reconocer a las mujeres como protagonistas de prácticamente ninguno de los temas estudiados a lo largo del grado causa perplejidad entre el alumnado, especialmente entre las estudiantes, mostrándose incluso indignadas:

Algunas chicas nos preguntamos: '¿y las mujeres dónde quedan?'. No lo sé, en el temario no están (Chica, $3 \% 4^{\circ}$ curso, mixto).

El otro día en clase pensé: 'solo estamos estudiando la historia de los hombres'. En el caso de las mujeres es: 'ah sí, las mujeres y los niños', como algo secundario. Somos muchas mujeres, el $50 \%$ de la población... Echo mucho en falta estudiar qué ha pasado con las mujeres a lo largo de la historia (Chica, $3^{\circ} / 4^{\circ}$ curso, mixto).

Las estudiantes también denuncian la invisibilidad de las mujeres en el uso de ejemplos utilizados para ilustrar las explicaciones. La comunicación oral y escrita va más allá de palabras e imágenes, ya que construye imaginario simbólico, en este caso de quién es protagonista en la política:

Cuando se ponen ejemplos, sale 'un hombre...' Nunca está el ejemplo de una mujer. Por eso lo tenemos tan interiorizado (Chica, $3^{\circ} / 4^{\circ}$ curso, mixto). 
Siempre, si hay que poner un ejemplo así rápido, dicen 'un hombre va a no sé cuántos...' (Chica, $2^{\circ}$ curso, no mixto).

En general, no solo se invisibiliza a las mujeres, sino al propio género como construcción social, así como su impacto en las estructuras sociales, económicas o políticas. Además, como critica el alumnado, en la mayor parte de las asignaturas se siguen confundiendo las variables 'sexo' y ‘género', debido a la falta de formación del profesorado en perspectiva de género. Se tiende meramente a controlar por la primera variable, sin analizar las causas que hacen del hecho biológico de ser hombre o mujer una ventaja o desventaja en el acceso a recursos y oportunidades. En resumidas cuentas, el género no suele emplearse como categoría analítica:

Cualquier cosa sobre género, si es que lo hemos tocado, lo que hemos hecho es aprender a contabilizarlo (Chico, $2^{\circ}$ curso, mixto).

Cuando tratamos este tema, se acaba culpabilizando a los medios de comunicación, al sistema o a la educación. Te acaban dando los cuatro elementos que explican estas diferencias, pero sin entrar en el fondo de la cuestión (Chica, $2^{\circ}$ curso, mixto).

Esto conduce a que el alumnado, tanto chicos como chicas, tienda a concebir las desigualdades como un elemento del pasado. Aunque a medida que avanza la conversación aparecen elementos relacionados con el mundo laboral o la distribución de los cargos de responsabilidad política, la esfera privada está prácticamente ausente como fuente de desigualdad y de poder en su análisis. Esto refleja la rígida separación entre vida pública y vida privada que sigue predominando en el currículum de la Ciencia Política, como hemos subrayado en anteriores apartados:

Hemos nacido en una época en que representa que todo está más cambiado. Quiero decir, es impensable que sucedan discriminaciones de género como en la época de Franco. Pero en cambio nuestras madres... (Chica, $2^{\circ}$ curso, no mixto).

En algunos ámbitos sí hay desigualdad todavía, pero ni en clase, ni en la universidad, ni en ningún sitio; por lo menos yo no lo he notado (Chica, $2^{\circ}$ curso, no mixto)

Los discursos post-feministas que consideran la discriminación como algo ya superado tienden a estar muy extendidos entre el profesorado (Verge, Ferrer-Fons y González 2018) y el alumnado (Webber, 2005). Si la desigualdad no se problematiza, especialmente en los primeros cursos, se transmite la idea de que ya no existe. Como pone en evidencia este fragmento de la discusión, no puede ser responsabilidad del alumnado (re)conocer la relevancia del género sin haberlo trabajado en clase:

Yo no sé mucho del tema, pero tampoco lo veo algo muy esencial, porque no hay mucha diferencia, ¿no? (Chica $1,2^{\circ}$ curso, no mixto).
'No hay mucha diferencia' porque no te habías parado a pensarlo, pero ahora que empiezas a pensarlo a lo mejor ves que sí hay más diferencias (Chica 2 , $2^{\circ}$ curso, no mixto).

Es que, claro, no sé lo que no sé... (Chica $1,2^{\circ}$ curso, no mixto).

Resulta necesario, pues, que los programas de formación continua de las universidades ofrezcan cursos sobre perspectiva de género para que el profesorado pueda adquirir esta competencia. El alumnado percibe la ceguera al género en los contenidos como un déficit formativo grave que condiciona su capacidad de pensamiento crítico y reclama incorporar la perspectiva de género, de manera transversal, en el contenido de las diferentes asignaturas e incrementar la oferta formativa especializada:

Durante la carrera no se nos ha invitado a la crítica ni a la reflexión. Creo que esto es grave, la verdad (Chico, $2^{\circ}$ curso, mixto).

Hay temas que los repetimos y repetimos y hay otros que ni siquiera los hemos visto (Chica, $3^{\circ} / 4^{\circ}$ curso, mixto).

Creo que es un tema tan fundamental y vital para nuestro futuro como sociedad que debería haber una asignatura solo de desigualdad de género (Chico, $2^{\circ}$ curso, mixto).

La ceguera al género del currículum impacta también en los trabajos de asignatura o de fin de grado. No solo se ignora este enfoque sino que, en la elección del tema, subyace la idea de que no cabe o no resulta necesario realizar un análisis con perspectiva de género de los temas centrales de la Ciencia Política. Asimismo, el escaso conocimiento del profesorado en esta materia, incluso en su sub-campo de especialización, produce una "falta de respuesta académica" (Dahlerup 2010: 89). Como comentaron varias estudiantes, cuando la pregunta de investigación trata algún tema de género, se suele producir una negociación frustrante con los supervisores sobre su relevancia o se delega la supervisión a profesoras expertas en género y política, aunque no sean especialistas en la temática principal del trabajo en cuestión.

\section{Los sesgos de género en el entorno de aprendizaje}

Todas las personas que interactúan en un aula tienen género y su experiencia varía en función del mismo, a partir de los estereotipos y normas sociales asociadas a la construcción social de ser hombre o mujer. El género no solo constituye una categoría social, sino también un proceso mediante el cual se reproducen las desigualdades y relaciones de poder (Beckwith 2005). En todos los grupos de discusión se señaló que los estudiantes participan más a menudo y con mayor seguridad que sus compañeras, sobre todo en las clases magistrales, las de mayor tamaño. Esto refleja y reproduce la desigualdad exis- 
tente en el espacio público, hecho que suele pasar desapercibido para el profesorado, según se destacó en los grupos de discusión:

Los chicos tienen más seguridad. Las chicas, por lo menos yo, en una clase magistral nunca diré nada (Chica, $2^{\circ}$ curso, no mixto).

¿Quién levanta primero la mano? No lo hace casi nunca una chica, por no decir nunca (Chico, $2^{\circ}$ curso, mixto).

No hemos visto jamás ningún profesor hacernos conscientes de que hay dos grupos, de que hay diferencia de género (Chico, $2^{\circ}$ curso, mixto).

El profesorado debe reflexionar sobre cómo fomentar una participación igualitaria en el aula, de manera que tanto unos como otras desarrollen toda su potencialidad, tratándose de un ágora donde se adquieren y ponen en práctica habilidades comunicativas y argumentativas. Así pues, se trata de un aspecto a ser incluido también en la formación continua del profesorado. Sin identificar que este pánico escénico se nutre de los roles asignados socialmente a mujeres y hombres, como la infra-confianza con la que se sigue educando a las mujeres y la sobre-confianza que se inculca a los hombres, el aula deviene un reflejo de la desigualdad imperante y la ausencia de intervención la reproduce:

Al principio estaba muy cortada. $Y$ sé que era porque los chicos tienen facilidad para hablar en clase y las chicas... El hecho de pensar 'si hago esta pregunta pareceré una tonta que no ha entendido nada'. Muchas veces es porque todavía no tienes tu grupo de amigos o simplemente no conoces a la gente (Chica, $3^{\circ} / 4^{\circ}$ curso, mixto).

Las chicas se acercan más a preguntar al profesor, porque estás el profesor y tú, y simplemente no es ante toda la clase. Lo ves en las pausas: siempre que hay alguien hablando con el profesor es una chica, son colas de chicas (Chica, $2^{\circ}$ curso, mixto).

Otro factor inhibidor de la participación de las estudiantes es la interrupción que sufren a menudo por parte de sus compañeros, sin que el profesorado suela intervenir para evitarlo:

Me he sentido tantas veces pisada por el típico que tiene la voz más fuerte... Esto lo considero discriminación porque después habla otra persona, que por casualidades de la vida es un chico, y no hay esta facilidad para cortarle la palabra; notas que te falta al respeto (Chica, $3^{\circ} / 4^{\circ}$ curso, mixto).

Los chicos tienen mucho más impulso a hablar y cortan. Se cortan entre ellos también, pero a las chicas se las corta casi siempre (...). La mayoría de las chicas no responden a este impulso sino que prefieren pasar del debate. Creo que esta clase de actitudes, si no somos capaces de gestionarlas por nosotros mismos, que se ve que no, los profesores son los que tendrían que decir... (Chica, $2^{\circ}$ curso, mixto).
Asimismo, el alumnado señaló que, en los trabajos grupales, las estudiantes suelen llevar el peso de las tareas más invisibles (como coordinación, organización o calendarización), mientras que los estudiantes hacen las presentaciones orales en clase. De nuevo, la mayor visibilidad en los roles de carácter más público es asumido por los hombres:

Teníamos que redactar un ensayo o bien prepararnos un tema y exponerlo ante toda la clase. Todos los que expusieron fueron chicos (Chica, $2^{\circ}$ curso, no mixto).

A veces, quizás sí que cogía el rol este de liderar, pero también porque las chicas de mi grupo me decían 'no, no, esto lo presentas tú' (Chico, $3^{\circ} / 4^{\circ}$ curso, mixto).

En cuanto al lenguaje, el alumnado considera que el profesorado no lo utiliza de manera inclusiva en sus clases. La mayoría de estudiantes no se había planteado nunca esta cuestión, aunque de la deliberación concluían que el lenguaje, como instrumento de transmisión del conocimiento, más allá del desdoblamiento de las formas masculinas y femeninas, arrastra sesgos de género en los ejemplos y las metáforas utilizados:

Si se nos habla durante dos horas de la 'ciudadanía', yo cuando escriba no voy a poner 'los ciudadanos' (Chica, $2^{\circ}$ curso, no mixto).

Más que cambiar el artículo deberíamos cambiar el contenido. No hablar de la 'mujer de la limpieza' sino del 'servicio de limpieza' (Chico, $2^{\circ}$ curso, mixto)

\section{Conclusiones}

La inclusión de la perspectiva de género en la docencia, en el marco normativo de la educación superior, no ha comportado la aplicación y supervisión efectivas por parte de las universidades y de las agencias de evaluación, respectivamente, observándose también en este campo las resistencias a la implementación efectiva de las políticas de igualdad. El análisis de la disciplina de la Ciencia Política revela que el género como categoría analítica transversal y las mujeres como protagonistas del conocimiento están esencialmente ausentes del currículum oficial de los grados, como muestra la escasez de competencias de género incluidas en las guías docentes, la baja inclusión de referencias a las desigualdades o la infra-representación de las mujeres en la bibliografía. Los manuales introductorios, por su parte, tanto los más antiguos como los más recientes, tampoco han transversalizado la perspectiva de género en sus contenidos, reproducen sesgos en los ejemplos empleados e invisibilizan tanto a las mujeres como actores políticos como a las obras producidas por politólogas, especialmente aquellas contribuciones que hacen un uso analítico y no meramente descriptivo de la variable 'género'. 
A su vez, los grupos de discusión nos han permitido explorar el impacto que tal ceguera tiene sobre el alumnado. La brecha de género en las referencias bibliográficas contribuye a crear un imaginario simbólico en que la autoridad científica es constitutivamente masculina, lo que incluso parece conllevar que el alumnado conceda menor autoridad a las profesoras. Asimismo, al no problematizarse la desigualdad ni trabajarse en clase contenidos relacionados con el género, el alumnado adopta la falacia de la igualdad o confía en la evolución del tiempo para lograrla: si no se hace visible un problema, no existe. Finalmente, la desigualdad tampoco resulta ajena al entorno de aprendizaje, reproduciéndose las lógicas de género en las interacciones en el aula y en los trabajos en grupo, sin que el profesorado intervenga de manera activa para motivar la participación de las estudiantes o evitar las frecuentes interrupciones que sufren.

Nuestra investigación sugiere que la ausencia de perspectiva de género del currículum puede estar limitando seriamente el desarrollo del pensamiento crítico del alumnado e incapacitando a graduados y graduadas para atender a las necesidades y expectativas del conjunto de la población en su futura práctica profesional. Por ejemplo, el hecho de no prestar atención a las dimensiones privadas de la desigualdad, dificulta la comprensión del alumnado sobre las estructuras, los procesos y los resultados políticos que resultan cruciales para políticas públicas clave como las relaciones familiares, las relaciones laborales, el bienestar o las violencias (Foster et al. 2013: 580). En definitiva, la transversalidad de la perspectiva de género en el currículum permitiría mejorar la calidad de la docencia y desarrollar la capacidad de contribuir al cambio social (Nuño Gómez y Álvarez Conde 2017: 294), entroncando con la tradición histórica de la Ciencia Política de combatir las injusticias flagrantes, como es el caso de la desigualdad entre mujeres y hombres (Matthes 2013: 238).

Los resultados de este estudio subrayan la necesidad de seguir analizando los distintos aspectos de la política docente afectados por sesgos de género, tales como las prácticas de contratación y promoción, las desigualdades en las carreras docentes o el tipo de formación docente que se imparte desde las universidades, así como de plantear soluciones para superarlos. También cabe analizar si los programas de postgrado adolecen también de esta ceguera o presentan mejores resultados. Finalmente, hacemos un llamamiento a identificar los mecanismos, incentivos y recursos necesarios para implicar al profesorado, a los centros y a las agencias de evaluación en la implementación efectiva de la transversalidad.

\section{Agradecimientos}

Parte de esta investigación fue financiada por el Center for Learning Innovation and Knowledge (CLIK) de la Universitat Pompeu Fabra (ref. 9PLAQUID 2013-2014) y contó con la participación de M. José González y Mariona Ferrer-Fons. El trabajo se enmarca dentro del Proyecto Unigual financiado por el Ministerio de Ciencia, Investigación e Universidades (Fem2017-86004-R). La contribución de Alba Alonso cuenta además con el apoyo de las Ayudas para la Consolidación y Estructuración de Grupos de Investigación de la Xunta de Galicia (Ref.ED431B 2017/47).

\section{NOTAS}

1. Para obtener información más detallada sobre estas cuestiones, véase el monográfico "La (in)visibilidad de las mujeres en la Educación Superior: retos y desafíos en la Academia" de la Revista Feminismos (29, 2017). Sobre las desigualdades de género en la carrera académica dentro de la Ciencia Política, véanse los monográficos de Politics \& Gender $(10: 3,2014)$ y PS: Political Science and Politics (48:3, 2015).

2. El listado de los 18 grados analizados se proporciona en el apéndice.

3. La excepción reciente es AQU Catalunya. Esta agencia, a través del Marco general para la introducción de la perspectiva de género en la docencia universitaria, ha establecido criterios de género en el proceso de verificación, seguimiento, modificación y acreditación de las titulaciones, dando cumplimiento al artículo 28 de la Llei 17/2015, d'igualtat efectiva de dones i homes.
4. No incluimos un indicador sobre objetivos y resultados de aprendizaje por la falta de información sobre los mismos en un volumen importante de guías docentes.

5. Véase https://www.upf.edu/xifres.

6. Se realizó un grupo solo con chicas para comprobar si los discursos y las dinámicas de la discusión se veían afectados por la participación de estudiantes hombres. No se observaron diferencias significativas con respecto al grupo mixto.

7. Las sub-secciones que tratan estos temas no fueron redactadas por Sodaro, sino por dos colaboradoras, como se menciona en los agradecimientos del manual.

8. Véase https://academic.oup.com/isq/pages/General_ Instructions.

9. Véase: https://jlsumner.shinyapps.io/syllabustool. 


\section{Referencias Bibliográficas}

Alonso, A. 2015. El mainstreaming de género en España. Tirant lo Blanch: Valencia.

Alonso, A. y E. Lombardo. 2016. "Ending ghettoization? Mainstreaming gender in Spanish political science education". European Political Science 15 (3): 292-302. https://doi.org/10.1057/eps.2015.77

ANECA. 2005. Libro Blanco. Título de Grado en Ciencias Políticas y de la Administración, Sociología y Gestión y Administración Pública. Madrid: ANECA.

Arbuckle, J. y B. D. Williams. 2003. "Students' perceptions of expressiveness: Age and gender effects on teacher evaluations". Sex Roles 49 (9/10): 507-516. https:// doi.org/10.1023/a:1025832707002

Atchison, A. L. 2013. "The practical process of gender mainstreaming in the political science curriculum". Politics \& Gender 9 (2): 228-235. https://doi.org/10.1017/ s1743923x13000081

Atchison, A. L. 2017. "Where are the women? An analysis of gender mainstreaming in introductory political science textbooks". Journal of Political Science Education 13 (2): 185-199. https://doi.org/10.1080/15512169.2017. 1279549

Bates, S., L. Jenkins y Z. Pfaelger. 2012. "Women in the profession: The composition of UK Political Science departments by sex". Politics 32 (3): 139-152. https://doi. org/10.1111/j.1467-9256.2012.01444.x

Barreda, M. y L. M. Ruiz (coords.). 2016. Análisis de la política. Enfoques y herramientas de la Ciencia Política. Barcelona: Huygens

Beckwith, K.. 2005. "A Common Language of Gender". Politics and Gender 1 (1): 128-137. https://doi.org//10.1017/ S1743923X05211017

Bosque, I. 2012. "Sexismo lingüístico y visibilidad de la mujer". Boletín de Información Lingüística de la Real Academia Española (1): 1-18. http://revistas.rae.es/bilrae/ article/view/120

Bustelo, M. 2014. "Evaluación de políticas públicas con perspectiva de género". Pp. 189-216 en Ciencia política con perspectiva de género, coordinado por M. Lois y A. Alonso. Madrid: Akal.

Cassese, E. C. y A. L. Bos. 2013. "A Hidden Curriculum? Examining the Gender Content in Introductory-Level Political Science Textbooks". Politics \& Gender, 9 (2): 214223. https://doi.org/10.1017/S1743923X13000068

Castaño, C., S. Vázquez-Cupeiro y J. L. Martínez-Cantos. 2017. "Gendered management in Spanish universities: functional segregation among vice-rectors". Gender and Education. https://doi.org/10.1080/09540253.2017.1410109

Cavaghan, R. 2017. "Bridging rhetoric and practice: New perspectives on barriers to gendered change". Journal of Women, Politics \& Policy 38 (1): 42-63. https://doi.org /10.1080/1554477X.2016.1198209

Colomer, J. M. 2017. Ciencia de la política. Madrid: Ariel

Comisión Europea. 2000. Science policies in the European Union: Promoting excellence trough mainstreaming gender equality. A Report from the ETAN Expert Working Group on Women and Science. Brussels: DG Research.

Consejo de Europa. 1998. Conceptual framework, methodology and presentation of good practices: Final report of activities of the Group of Specialists on Mainstreaming. EGS-MS. Estrasburgo: Consejo de Europa.

Dahlerup, D. 2010. "The development of gender and politics as a new research field within the framework of the ECPR". European Political Science 9: S85-S98. https://doi.org/10.1057/eps.2010.45

Del Águila, R. (ed.). 1997. Manual de Ciencia Política. Madrid: Trotta.

Elizondo, A. 2015. "The status of women in Spanish Political Science." European Political Science 14 (2): 96-104. https://doi.org/10.1057/eps.2015.6

Ferree, M. M. y E. J. Hall. 1996. "Rethinking Stratification from a Feminist Perspective: Gender, Race, and Class in Mainstream Textbooks". American Sociological Review 61 (6): 929-950. https://doi. org/10.2307/2096301

Foster, E., P. Ker, A. Hopkins, C. Byrne y L. Ahall. 2013. "The Personal is Not Political: At Least in the UK's Top Politics and IR Departments". British Journal of Politics \& International Relations 15 (4): 566-585. https://doi. org/10.1111/j.1467-856X.2011.00500.x

Grünberg, L. 2011. "From gender studies to gender IN studies and beyond". En L. Grünberg (ed), From gender studies to gender IN studies. Case studies on genderinclusive curriculum in higher education. Bucharest: Unesco - CEPES.

Hakanson, M. 2005. "The impact of gender on citations: An analysis of College \& Research Libraries". Journal of Academic Librarianship, and Library Quarterly College \& Research Libraries 4 (66): 312-322. https://doi. org/10.5860/crl.66.4.312

Izquierdo, M. J. [dir.]. 1999. El sexisme a la universitat. Estudi comparatiu del personal assalariat de les universitats públiques catalanes. Barcelona: Servei de Publicacions de la Universitat Autònoma de Barcelona.

Izquierdo, M. J., F. J. León y E. Mora. 2008. "Sesgo de género y desigualdades en la evaluación de la calidad académica". Arxius de Ciències Socials 19: 75-90. http:// roderic.uv.es/handle/10550/19426

Kantola, J. 2008. '“Why do all the women disappear?' Gendering processes in a political science department". Gender, Work and Organization 15 (2): 202-225. https:// doi.org/10.1111/j.1468-0432.2007.00376.x

Kortendiek, B. 2011. "Supporting the Bologna Process by gender mainstreaming: A model for the integration of gender studies in higher education curricula." Pp. 212-228 en From Gender Studies to Gender IN Studies. Case Studies on Gender-Inclusive Curriculum in Higher Education, editado por L. Grünberg. Bucharest: Unesco CEPES.

Lois, M. y A. Alonso (coords.). 2014a. Ciencia política con perspectiva de género. Madrid: Akal.

Lois, M. y A. Alonso. 2014b. "Instituciones y estructuras políticas". Pp. 69-97 en Ciencia política con perspectiva de género, coordinado por M. Lois y A. Alonso. Madrid: Akal.

Lois, M. y M. de la Fuente. 2014. "Teoría política". Pp. 39-68 en Ciencia política con perspectiva de género, coordinado por M. Lois y A. Alonso. Madrid: Akal.

Lombardo, E. y L. Mergaert. 2013. "Gender mainstreaming and resistance to gender training. A framework for studying implementation". NORA - Nordic Journal of Feminist and Gender Research 21 (4): 296-311. https:// doi.org/10.1080/08038740.2013.851115

Lovenduski, J. 1998. "Gendering research in political science". Annual Review of Political Science 1: 333-356. https:// www.annualreviews.org/doi/full/10.1146/annurev.polisci.1.1.333 
Lyle-Gonga, M. 2013. "A critical analysis of gender mainstreaming". Politics \& Gender 9 (2): 209-213. https://doi. org/10.1017/S1743923X13000056

MacNell, L., A. Driscoll y A. N. Hunt. 2015. "What's in a name: Exposing gender bias in student ratings of teaching". Innovative Higher Education 40 (4): 291-303. https:// doi.org/10.1007/s10755-014-9313-4

Maliniak, D., R. Powersa y B. F. Walter. 2013. "The gender citation gap in International Relations". International Organization 67 (4): 889-922. https://doi.org/10.1017/ S0020818313000209

Martínez Hernández, E. 2014. "Las formas de acción colectiva: partidos políticos y movimientos sociales" Pp. 129160 en Ciencia política con perspectiva de género, editado por M. Lois y AAlonso (coords.). Madrid: Akal.

Mason, M. A. y M. Goulden. 2004. "Marriage and baby blues: Redefining gender equity in the academy". Annals of the American Academy of Political and Social Science 596 (1): 86-102. https://doi. org/10.1177/0002716204268744

Matthes, M. 2013. "Conclusion and Rejoinders". Politics \& Gender 9 (2): 235-238. https://doi.org/10.1017/ S1743923X13000093

Mertus, J. 2007. "Teaching Gender in International Relations". International Studies Perspectives 8 (3): 323-325. https://doi.org/10.1111/j.1528-3585.2007.00295.x

Monroe, K., S. Ozyurt, T. Wrigley y A. Alexander. 2008. "Gender equality in academia: Bad news from the trenches and some possible solutions". Perspectives on Politics 6 (2): 215-232. https://doi.org/10.1017/ S1537592708080572

Mügge, L., E. Evans y I. Engeli. 2016. “Introduction: Gender in European Political Science Education - Taking stock and future directions". European Political Science 15 (3): 281-291. https://doi.org/10.1057/eps.2015.72

Nuño Gómez, L. y E. Álvarez Conde. 2017. “Androcentrismo académico: La ficción de un conocimiento neutral". Revista Feminismos 29: 279-297. https://doi. org/10.14198/fem.2017.29.11

Peterson, E., S. López y R. [Lucas] Platero. 2014. "Políticas públicas." Pp. 162-187 en Ciencia política con perspectiva de género, editado por Marta Lois y Alba Alonso (coords.). Madrid: Akal.

Rifà-Valls, M. y L. Duarte. 2013. "Interseccionalidades del género, desigualdad y educación superior. Categorías, indicadores y estrategias en la investigación". Pp. 77-97 en La interseccionalidad en debate. Actas del Congreso Internacional Indicadores interseccionales y medidas de inclusión social en instituciones de educación superior (23-27 Nov 2012), editado por M. Zapata, S. García y J. Chan. Berlín: Instituto de Estudios Latinoamericanos. http://www.upla.cl/inclusion/ wp-content/uploads/2015/04/Interseccionalidadendebate_misealweb-1.pdf

Schmidt, B. 2015. Gendered language in teacher reviews. Recurso en línea. Última consulta 15 de septiembre de 2017 (http://benschmidt.org/2015/02/06/rate-myprofessor).

Sodaro, M J. 2006. Política y Ciencia Política. Una introducción. Madrid: MacGraw-Hill.

Stake, R.E. 1999. Investigación con estudios de caso. Madrid: Ed. Morata.

Timperley, C. 2013. "Women in the Academy: Key studies on gender in political science". Political Science 65 (1): 84-104. https://doi.org/10.1177/0032318713489783

Tolleson-Rinehart, S y S. Carroll. 2006. "Far from ideal': The gender politics of political science". American Political Science Review 100 (4): 507-513. https://doi. org/10.1017/S0003055406062368

Uriarte, E. 2010. Introducción a la Ciencia Política. La política en las sociedades democráticas. Madrid: Tecnos.

Valiente, C. 2002. "An overview of research on gender in Spanish society". Gender and Society 16 (6): 767-792. https://doi.org/10.1177/089124302237887

Vallès, J. M. 2000. Ciencia Política: Una introducción. Madrid: Ariel.

Verge, T. 2014. "Comportamiento político". Pp. 99-128 en Ciencia política con perspectiva de género, coordinado por M. Lois y A. Alonso. Madrid: Akal.

Verge, T. y T. Cabruja. 2017. La perspectiva de gènere en docència i recerca a les universitats de la Xarxa Vives. Situació actual i reptes de futur. Xarxa Vives: Castelló de la Plana [http://vives.org/PU3.pdf].

Verge, T., M. Ferrer-Fons y M. J. González. 2017. Informe la perspectiva de gènere en la docència, Grau en Ciències Polítiques $i$ de l'Administració. Barcelona: Universitat Pompeu Fabra [https://www.upf.edu/ documents/7195201/200373641/CPIS_2014.pdf/ cae8050e-473d-e715-dcc8-fa65cdb40c51].

Verge, T., M. Ferrer-Fons y M. J. González. 2018. "Resistance to mainstreaming gender into the higher education curriculum". European Journal of Women's Studies 25 (1): 86-101. https://doi.org/10.1177/1350506816688237

Verloo, M. (ed.). 2018. Varieties of Opposition to Gender Equality in Europe. London: Routledge.

Wahlke, J. C. 1991. "Liberal Learning and the Political Science Major: A Report to the Profession". PS: Political Science \& Politics 24 (1): 48-60. https://doi. org/10.2307/419376

Waylen, G., K. Celis, J. Kantola y S. L. Weldon (eds). 2013. Oxford Handbook of Gender and Politics. Oxford: Oxford University Press.

Webber, M. 2005. “'Don't be so feminist': Exploring student resistance to feminist approaches in a Canadian university". Women's Studies International Forum 28 (2-3): 181-194. https://doi.org/10.1016/j. wsif.2005.04.006

Wenneràs, C. y A. Wold. 1997. "Sexism and nepotism in peer review". Nature 387: 321-343. https://doi. org/10.1038/387341a0

TÀNIA VERGE MESTRE es profesora contratada doctora en el Departamento de Ciencias Políticas y Sociales de la Universitat Pompeu Fabra. Su investigación se centra principalmente en el género y la política, la representación y los partidos políticos, así como las resistencias a la adopción y la implementación de las políticas de igualdad.

ALBA ALONSO ÁLVAREZ es profesora ayudante doctora en el Departamento de Ciencia Política y Sociología de la Universidade de Santiago de Compostela. Su ámbito principal de especialización hace referencia al género y la política, con especial atención a la implementación de políticas de igualdad, el estudio de la distribución territorial del poder desde la perspectiva de género y el análisis del movimiento feminista. 